\section{Automatic Ex-post Flood \\ Assessment Using Long Time \\ Series of Optical Earth \\ Observation Images}

Gl_Forum 2017, Issue 1

Page: 217 - 227

Full Paper

Corresponding Author: martin.sudmanns@sbg.ac.at

DOI: 10.1553/giscience2017_01_s217

\author{
Martin Sudmanns' ${ }^{1}$ Dirk Tiede 1 , Lorenz Wendt' and Andrea Baraldi1,2 \\ 'Department of Geoinformatics - Z_GIS, University of Salzburg, Austria \\ 2Department of Agricultural and Food Sciences, University of Naples Federico II, Italy
}

\begin{abstract}
Our study uses a dense temporal stack of 78 Landsat 8 images for surface water extraction using automatic Earth Observation (EO) image pre-processing, coupled with analyses over time for flood detection. The analysis is conducted with our IQ (ImageQuerying) system developed in-house, which allows ad-hoc executing of spatio-temporal queries against semantically enriched EO images. To facilitate high performance analyses, the data are stored as a spatio-temporal data cube in an array database. The analyses are automatically-translated database queries, which increase reproducibility, readability and comprehensibility for a human operator and can be conducted within just a few minutes. The specific analysis for this contribution is based on flood-extent mapping over different user-definable time spans. The results indicate areas that have been flooded at least once in the selected time span and are therefore prone to being flooded in future events. Additional spatial queries (e.g., for the indication of cloud cover) support the quality assessment of the flood analyses. We compared our result with a flood mask derived from a SAR (synthetic aperture radar) image of a single event in Somalia (Hiran province). Larger flooded areas overlap in both analyses, despite the non-synchronous acquisition times of the images. The results can be used as input for improved risk assessment and management of floods.
\end{abstract}

\title{
Keywords:
}

remote sensing, time series, flood mapping, big Earth data, data cube

\section{Introduction}

A flood is a natural phenomenon that can be either beneficial or destructive. One of the most prominent examples of a beneficial flood is that of the River Nile, whose yearly floods help to fertilize the agricultural fields along its banks. However, generally increasing competition between land uses, pressure caused by expanding cities, and spreading agricultural areas are turning floods into undesired events that cause severe damage and loss of life (Plate 2002). Further, surface waters are sensitive to changes in global and local climate conditions (Hirabayashi et al., 2013), which makes the handling or prediction of 
floods difficult. Monitoring floods and surface waters in general by investigating their underlying spatio-temporal processes is crucial not only for better disaster management, but also for prevention of damage and of individual, personal negative impacts (Creutin et al., 2009). Our analysis uses regularly acquired EO (Earth Observation) images from the Landsat 8 satellite with the aim of supporting general flood management and temporal flood pattern analysis. Our analysis is not intended for emergency mapping purposes.

Several characteristics of floods motivate EO-image-driven analyses. (1) Continuous spatial dimension. The spatial extents of floods encompass large areas, making delineation using insitu observations difficult and challenging, especially when the flooding causes inaccessibility to the regions. EO images, however, cover larger areas and therefore allow a broader view of the event from a bird's eye perspective. (2) Continuous data acquisition. If the flooding is not part of a natural, recurrent and predictable phenomenon and is affecting human settlements, it is necessary to react quickly in order to provide timely information for disaster management. High-resolution (HR) EO images are acquired regularly, and in many cases independently of the presence or absence of specific and known events or phenomena. (3) Long-term temporal dimension. To investigate long-term changes of surface waters and flood trends, it is necessary to compare their characteristics over many years (including expost analyses). For example, to relate surface water trends to long-term climate changes, at least 30 years of continuous data collection would have to be targeted, as this is the standard reference period, defined by the World Meteorological Organisation (WMO). (4) Continuous semantic dimension. The inter-comparison of observations requires some implicit assumptions. For example, to increase the probability that radiometric changes observed by recurring satellites carrying imaging sensors in Space correspond to actual changes on the ground, the measurement and processing procedures must be the same. This includes factors such as sensor type, the acquisition time or the satellite orbit, and following pre-processing steps such as radiometric calibration to TOA (top-of-atmosphere) reflection, atmospheric correction to BOA (bottom-of-atmosphere) reflection, and topographic correction. Another example is the definition of a flood, including its semantics, and beginning and ending times. Comparing already existing flood masks of different study areas - in many cases across administrative borders - over a long time span is based on the assumption that the underlying definition of a flood does not vary across geographical location and time. Large EO image archives from Landsat or - in the future - Sentinel are reliable data resources for this type of application. With their long-term and continuous data collection, EO images are suitable to address the aforementioned requirements and assumptions for the monitoring of floods.

Traditionally, flood masks are extracted using imaging radar (SAR, synthetic aperture radar), because the low radar backscatter allows discrimination of the smooth water surface from the high backscatter of the surrounding environment (Ulaby et al., 2014, p. 23). Moreover, microwave radiation is able to penetrate clouds and even light rain. Therefore, SAR imagery is independent of the atmospheric condition. However, strong winds, which whirl up the water surface, and stronger precipitation influence the signal and may also result in high backscatter (Danklmayer et al., 2009). This paper describes an alternative approach that harvests archived optical EO images for any observed and recorded flood event as input for future flood management. All archived optical EO data for a certain area are pre-classified 
fully automatically into general-purpose semi-symbolic information layers. The information layers are generic and applicable to multiple domains. Thus, apart from a single database query, the flood mapping application presented here does not require any specific workflow or processing steps if the information layers are available prior to the analysis. The same information layers can be used in different contexts as well. This is a great benefit because any application can be set up on top of the data store by applying an ad-hoc, but re-usable, database query on completely different time intervals.

An analysis over larger areas might process GBs or even TBs of data, which needs to be completed within a reasonable time. If timely information is required or the processing capabilities are shared, the process of analysing the data has to be to be as fast as possible. To achieve sound scientific underpinning and to repeat the analysis for a different study area or at a later time, the analysis must be completely reproducible. Therefore, fully automated methods in a high-performance processing environment are desirable. In this study, the automated pre-classification of EO images in combination with a data-cube-based storage engine is utilized to achieve fast, comprehensible and reproducible results.

\section{Methods}

The study area is located in Hiran province in south-central Somalia, about $200 \mathrm{~km}$ north of the capital city Mogadishu. The Shebelle River (Somali: Webi Shabeelle), which rises in Ethiopia and flows southeast towards Mogadishu, is of particular importance in this study. A regular flooding of its banks characterizes the Shebelle River. In flat areas, such as in the study area, larger zones are flooded causing damage that is severe in places. A recent example is a severe flooding event in mid-May 2016 which resulted in more than 70,000 people being displaced from the region's main city, Belet Weyne (OCHA, 2016). These characteristics of the study area make it well suited for extracting the flooded areas using a long time series of optical EO images.

To demonstrate the technical feasibility of our approach, Landsat 8 images were used. Landsat 8 is the youngest member of the NASA/USGS family of satellites and carries the OLI (Operational Land Imager) optical imaging sensor to monitor the Earth's surface. Launched in 2013, it provides global monitoring every 16 days. All available images to date of the study area (path/row: 164/057) were downloaded from the USGS Earth Explorer in September 2016, regardless of their cloud cover. In total, 78 images, covering three years of acquisition, were used for the analyses. All images were pre-processed fully automatically into information layers using the SIAMTM software (Satellite Image Automatic Mapper).

The SIAMTM Software implements a spectral rule-based decision-tree classifier (Baraldi et al., 2010). SIAMTM takes any multispectral EO image and outputs information layers, such as a scene classification map (SCM) or a vegetation mask, in near real-time and parameterfree, i.e. without human interaction. The SCM is a discrete and finite set of semi-symbolic spectral categories, e.g. vegetation. Each SCM is segmented to allow queries using shape and size information. An automated workflow in eCognition aggregates adjacent pixels of the scene classification map with the same semantic label into connected objects. This workflow calculates the size and shape information for each object. In the case presented, the 
compactness parameter is used (length/width divided by the area). This is a measure of how similar the object is to a rectangle as the most perfect form and subsequently allows reasoning about the object's shape complexity. It is re-coded into raster cells as information layers to support additional queries. Size and shape are concepts that increase the explanatory power of further analyses. For example, the information allows the selection of only those classified areas that are of a certain size; the shape information allows the distinction between rivers and lakes.

The analyses over time on the available 78 Landsat 8 images (covering $\sim 3$ years) was conducted in IQ (ImageQuerying), an incrementally learning, web-based solution for analyses of massive EO image data sets (big Earth data). Developed in-house, it features both image-understanding capabilities using computer vision techniques and $\mathrm{EO}$ imageprocessing directly at the database level (Tiede et al., 2016). The available SCMs and derived shape information layers were batch-imported into IQ using the transactional Web Coverage Service (WCS-T). Internally, IQ creates multiple data cubes by stacking the images ordered by their temporal dimension (Sudmanns et al., 2016), utilizing the Rasdaman array database management system (DBMS) (Bauman et al., 1998). Analyses in IQ use one or more segmented SCMs, i.e. information layers, as a-priori knowledge and treat the results as posterior, which can be utilized as a-priori knowledge for further analyses.

The analyses use the pre-classified sub-symbolic spectral categories (semi-concepts) as apriori knowledge in IQ to extract surface water and flooded areas. Additional queries like cloud cover assessment are possible using the same information layers (based on different spectral categories) to support the quality assessment of the flood maps. The queries are implemented using the $\mathrm{n}$-ary operator condense (i.e. an operator with dynamic $\mathrm{n}$ input elements, where in this case $\mathrm{n}$ is the number of images) with an OR condition, as specified in the OGC Web Coverage Processing Service (WCPS) for all pixels labelled as one of the classes showing a spectral signature similar to sub-symbolic water-like categories (e.g. turbidwater like or deep-water like). More specifically, the operator iterates through every $\{x, y\}-$ location of the study area with $\mathrm{x}$ and $\mathrm{y}$ as the pixel positions of the images. The pixels at every $\{\mathrm{x}, \mathrm{y}\}$-location through all images make a time series of categorical variables. The condense function, parameterized with OR, takes the whole time series as input and labels it $\{1\}$ if at least one element of the time series fulfils the criterion specified by the labels of the sub-symbolic spectral categories (e.g., category 21 (turbid-water like) or 22 (deep-water like)). Otherwise, it is labelled $\{0\}$. By way of comparison, a parameterization with AND would label the time series with $\{1\}$ if all of its elements fulfil the criterion. Therefore, the approach treats clouds, for example, as not water implicitly. Mapping all of the labelled time series back to a single, multi-temporal 2D-image results in a binary map that shows which pixels were classified as water at least once within the selected time span. The results were not manually corrected for errors.

The analysis was conducted on a virtual machine running RHEL 7 (Red Hat Enterprise Linux). This machine operates a single stand-alone node of the Rasdaman array-DBMS. It is equipped with 4 virtual CPUs with $2.50 \mathrm{GHz}$ clocking and $16 \mathrm{~GB}$ virtual RAM. The data storage is located on common, virtual, all-use storage. No dedicated high-performance storage system was utilized for this feasibility study. 
The result of the main analysis (extraction of flooded area in the first half of 2016) is compared with another automatic approach using a SAR image from a single timestamp (30.05.2016) in the middle of the time span for our analysis. The result of this analysis was kindly provided by the German Aerospace Center, DLR. The SAR-based flood mask was derived automatically by the Sentinel-1 Flood Service (S-1FS) developed at DLR (Twele et al., 2016), which is geared towards near real-time flood detection. S-1FS consists of a fullyautomated processing chain containing the following steps: automatic data ingestion, preprocessing of the EO data, computation and adaption of global auxiliary data (digital elevation models, topographic slope information, topographic indices, and reference water masks), unsupervised initialization of the classification, post-classification refinement, and dissemination of the crisis information via a web-client (Martinis et al., 2015).

\section{Results}

The results of the automated extraction of flooded areas alongside the Shebelle River are depicted in Figure 1. Input for the analyses consisted of the information layers of all available Landsat 8 images covering this area captured between January 2016 and early September 2016. The spatial extent of the subset covers an area of $7 \times 9 \mathrm{~km}$ with a pixel size of $30 \mathrm{~m}$. More than 5.1 million observations in space and time need to be accessed and processed within the ad-hoc query to conduct the analysis. The analysis took less than 10 seconds to conduct the database query on the non-cached data. 


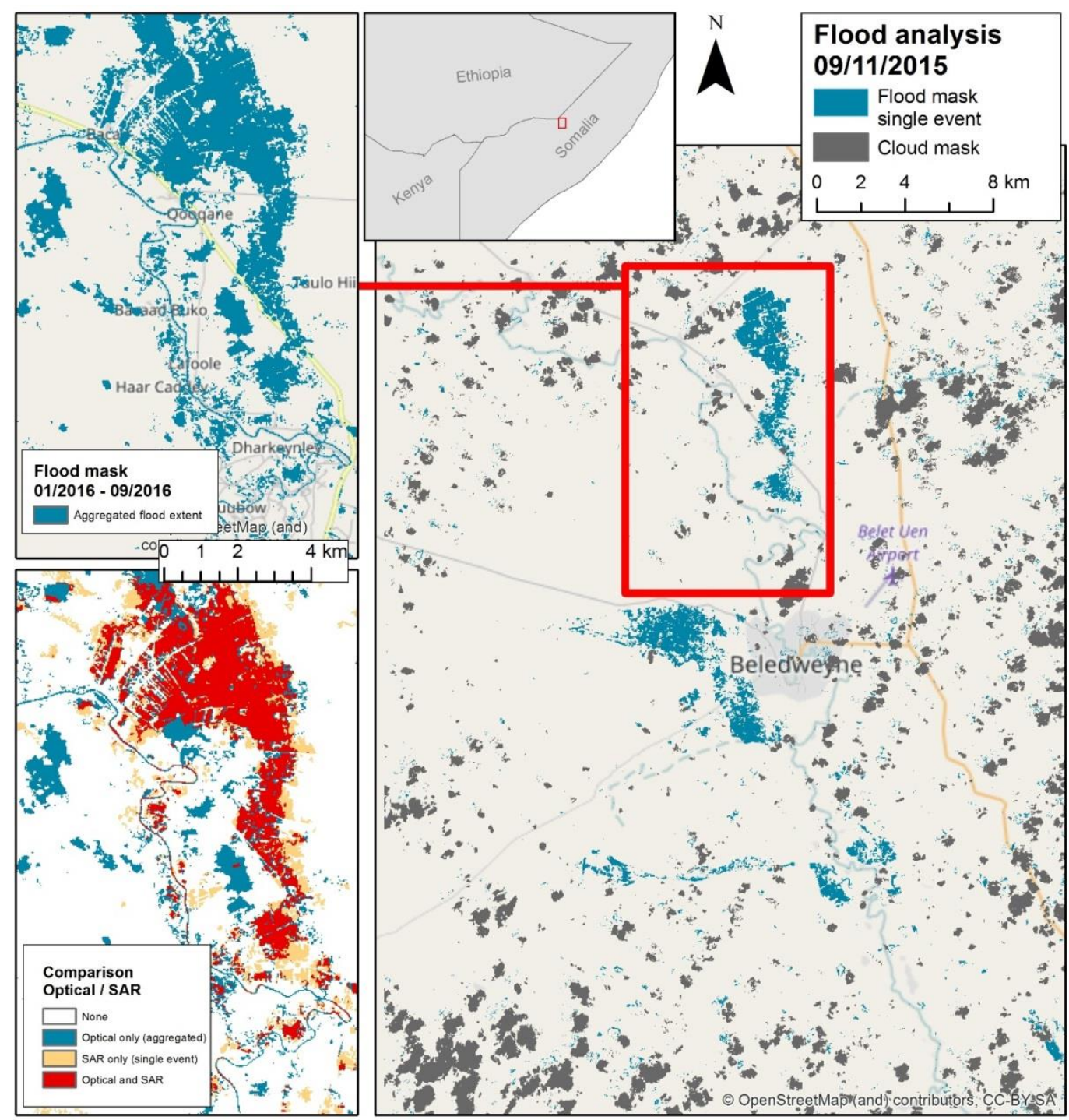

Figure 1: (a, top-left) Extracted flood mask using a long time series of optical EO images. (b, bottomleft) Differences between the flood mask generated using the optical images and the flood mask generated using the SAR image. The SAR image analysis was kindly provided by the German Aerospace Center (DLR). (c, right) Analysis of a single flood event in Oct./Nov. 2015. In addition to an extraction of the flood extent, a cloud mask was extracted based on spectral categories. All basemaps are copyrighted by OpenStreetMap contributors and available from https://www.openstreetmap.org/. The analysis output of the system has undergone cartographic preparation for visualization purposes.

In Figure 1 (a), the flooded areas, shown in blue, are clearly visible alongside the Shebelle River, which is visible as the long linear structure. Flooding was detected on the left and right sides of the river, especially in the northern parts (as recorded by Landsat 8 with its 16-days recurring acquisition plan). In this larger area, structural elements of the agricultural field 
systems are also visible. In the northern part of Figure 1 (a) and Figure 1 (b), three roads, perpendicular to the river, are distinctly visible as they were mostly not flooded due to their slightly higher elevation. Also distinctively visible are a large number of small areas, which are labelled as flooded, but have ragged borders, and some larger areas with smoother corners. This could indicate that they have different underlying spatio-temporal phenomena.

Differences between the results of the approach based on optical images and the SAR-based approach are shown in Figure 1 (b). The comparison of the time-series analysis with a single event based on the SAR image was conducted to give an indication of whether such a major event is also discernible in our approach. In both approaches, areas without the label water are shown in dark grey. They make up the majority of the result. Red areas have been identified as surface water by both approaches, whereas yellow areas are only identified in the SAR image, and blue areas are only identified by the long time series of optical EO images. Table 1 is a statistical representation of the images. While the majority of the pixels are labelled 'not water', the pixels labelled 'water' by both approaches represent $10.90 \%$ of the total number of pixels; the pixels labelled 'water' by the optical approach amounted to only $8.27 \%$; and the pixels labelled 'water' by the SAR approach represented $6.81 \%$. Another way to compare the two approaches is to check whether the main objects are identified. As shown in Figure 1 (b), both methods are able to identify the main flooding areas on the eastern side of the river, with some differences in exact delineation at the borders. Only the analysis of the optical EO images created larger areas without a corresponding match in the analysis of the SAR image, which in most cases reflects additional flooding events over time. The river itself is a connected and uninterrupted object in the result of the analysis of the optical EO images, while it is incomplete in the analysis of the SAR image. On the other hand, in the optical images, some areas that were identified as surface water might in fact be cloud shadows.

Table 1: Tabular representation of the differences.

\begin{tabular}{|l|l|l|}
\hline Class & Number of Pixels & Percentage \\
\hline Without Water & $1,161,580$ & $74.01 \%$ \\
\hline Optical Only & 129,818 & $8.27 \%$ \\
\hline SAR Only & 106,947 & $6.81 \%$ \\
\hline Optical and SAR & 171,111 & $10.90 \%$ \\
\hline Total & $1,569,456$ & $100.00 \%$ \\
\hline
\end{tabular}

Figure 1 (c) shows a second analysis of a Landsat 8 image shortly after a flood event in October/November 2015. In this example, the flood map is based on one image only (09/11/2015), which was identified by an initial query to show the maximum extent of the event. In addition, the surface water mask is accompanied by a cloud mask, which was also extracted based on the spectral categories assigned in the pre-processing steps. This helps in interpreting the quality of the flood mask, e.g., to identify areas which are not taken into account because they are below clouds. It also allows the identification of cloud shadows 
which were wrongly identified as 'water'. This probably happens because cloud shadows usually have the same spectral signature as water. In this case, they are small, blue-coloured areas showing the north-westerly direction of the clouds.

\section{Discussion}

It should be noted that the analysis focuses on regularly collected images, which are not captured explicitly for recognizing peaks of specific flooding events. The analysis is relevant for general flood management / flood pattern analysis through time but not for emergency mapping purposes. Further, the system is currently still in the early stages of development. Nevertheless, some benefits have already emerged.

The fully automated procedure in IQ, which allows 'any query, any time' with reasonable response times and without a long preparation time, has several advantages. It allows users to save time and start directly with the analysis without having to deal with data management issues. Further, it speeds up the analysis in general, since the EO images are pre-processed soon after they become available. Subsequently, users can extract information in near-real time and on demand for any given area of interest or time span. In contrast to other approaches, such as generating an explicit water mask using SAR imagery, this approach is based on multi-purpose information layers and allows re-using the same data for follow-up queries or completely different applications. This allows flexibility in the analysis and reduces processing time and storage requirements. The image-understanding component allows users to formulate queries based on their real-world knowledge and in 'user-speak' without having to deal with low-level system details. These characteristics distinguish it from other approaches.

The overall performance is promising for scaling up to larger analyses. Further improvements for the operational use could be implemented without development costs by using the capabilities of the array DBMS. For example, array DBMSs allow horizontal scaling by splitting up the data on multiple servers and therefore also dividing up the workload during the analyses. Since there is no data dependency across the individual time series analyses, the parallelization of this type of analysis is feasible. More important than the time required for processing is the time required for data preparation and defining the workflow. The approach we present assumes that the data is already within the database, fully automated, calibrated and pre-processed. This is the first significant difference to traditional workflows, where up to $50 \%$ of the time has to be spent on data management and preparation (Giuliani, Dubois \& Lacroix, 2013). An example of such a time-consuming process is the extraction of global water surface carried out by Pekel et al. (2016), who spent two years preparing the data prior to its analysis. The second difference is that an experienced and skilled operator can create the database query within a few minutes. The declarative approach hides numerous technical, data- and sensor-specific considerations from the analysist or automates them. Further, the query can be stored and shared in IQ's knowledge base. It is therefore possible to reuse the query directly for a new spatio-temporal subset. Although there is room for improvement to exploit the full potential of the array 
DBMS, the study showed that declarative querying of information layers can save a lot of time.

Since there is no ground-truth available yet, quality assessment is difficult. Nevertheless, the comparison with the water mask of a major event extracted from SAR imagery shows that the two water masks have similar characteristics, although one has to keep in mind that the masks generated using SAR imagery are not necessarily correct. They might overclassify water areas, e.g., due to smooth, compressed sand. Some differences between the two products can be explained by their different acquisition times, so that the flood peak is not perfectly covered, and by the merging of flooded areas from different dates in the optical approach, which include additional small local flooding events. The larger areas with smooth borders in particular might be cloud shadows misclassified as water. This is due to the current limitation of the automatic pre-processing, which sometimes labels dark cloud shadows as water because their spectral signatures are similar. The second example showed the possibility of using a cloud mask as a quality indicator for the result. For time series analysis, cloud/cloud shadow problems might be mitigated by including the context of the objects in the pre-processing step, for example if an improved contextual cloud/cloud shadow detection method is incorporated.

The aim of this study was to show the feasibility of the approach. Therefore, the database query was kept very simple and comprehensive, and further work is required to improve the query. Three improvements can be named here. Currently, clouds in the time series analysis are treated as no water and not as no data. While this relabelling could be achieved with moderate effort, addressing objects instead of pixels in the analysis is more complicated. This is necessary if a user wants to extract only those flooding areas of a certain size or wants to exclude the river from the flooded area. In the current implementation, additional postprocessing steps in a Geographic Information System are required. An uncertainty layer might be included, if it is provided together with the information layer. Another improvement could be to label how often a match was found in the time series, instead of the simple binary labelling. Some of these improvements are fairly straightforward to implement and will be addressed soon, while others require significantly more work and improvements of the overall IQ system.

To increase the relevance and significance of the analysis, it could be coupled with auxiliary data sets. In this feasibility study, the start and end times of the time series analysis were chosen pragmatically: the start time was set to 1 January 2016; the end time was determined by the availability of data. For example, signals coming from a water measuring point somewhere in the study area indicating the flooding event could be used as a dynamic starting point of the time series or trigger the map-creation process. Therefore, to support joining the in-situ data with the EO images, both have to be stored as OGC coverage in the array-DBMS. The long-term vision is that this can be done automatically and within the database, using a single query. 


\section{Conclusion}

Analysing surface water changes over time using high-resolution EO images yields several benefits for flood management. Areas which are at risk of flooding can be extracted by aggregating all pixels that have been flooded at least once within a certain time span. Such information is not usually available in common data sets and requires a lot of manual work. It has been shown that the design of the system has the potential for fast analysis over a temporal stack of images, and upscaling for larger data sets. As soon as the data acquisition allows analyses through multiple seasons, the proposed system will allow extracting the frequency, duration and time of floods as long-term trends. Furthermore, it will be able to answer the question of which lakes or rivers had a flooding event at the same time in a certain area. The proposed method allows such questions to be answered ad-hoc by using semantic querying against a database of large EO data sets. Higher temporal coverage, e.g., through the Copernicus Sentinel 2 MSI (Multi Spectral Instrument), will further support the approach, making it more likely to capture maximum flood extents and increasing the number of cloud-free images.

\section{Acknowledgement}

The research has received funding from the Austrian Research Promotion Agency (FFG) under the Austrian Space Application Programme (ASAP) within the project EO4HumEn+ (Extended EO-based services for dynamic information needs in humanitarian services; contract no: 854041), from the exploratory project SemEO (FFG-ICT, contract no: 855467), and from the Austrian science fund (FWF) through the Doctoral College GIScience (DK W1237-N23). We would like to thank colleagues from the German Aerospace Center (DLR) for producing the SAR-based reference flood mask.

\section{References}

Baraldi, A., Durieux, L., Simonetti, D., Conchedda, G., Holecz, F., \& Blonda, P. (2010). Automatic spectral rule-based preliminary classification of radiometrically calibrated SPOT-4/-5/IRS, AVHRR/MSG, AATSR, IKONOS/QuickBird/OrbView/GeoEye and DMC/SPOT-1/-2 imagery - Part I: System design and implementation, IEEE Trans. Geoscience Remote Sensing, 48(3), 1299-1325. doi: 10.1109/TGRS.2009.2032457.

Baumann, P., A. Dehmel, P. Furtado, R. Ritsch, \& Widmann, N. (1998). The multidimensional database system RasDaMan. Acm Sigmod Record, 27(4), 575-577. doi: 10.1145/276304.276386.

Creutin, J.D., Borga, M., Lutoff, C., Scolobig, A., Ruin, I., \& Créton- Cazanave, L. (2009). Catchment dynamics and social response during flash floods: the potential of radar rainfall monitoring for warning procedures. Meteorological applications, 16(1), 115-125. doi: 10.1002/met.128

Danklmayer, A., Doring, B.J., Schwerdt, M., \& Chandra, M. (2009). Assessment of Atmospheric Propagation Effects in SAR Images. IEEE Transactions on Geoscience and Remote Sensing, 47(10), 3507-3518. doi: 10.1109/TGRS.2009.2022271. 
Giuliani, G., Dubois, A., \& Lacroix, P. (2013). Testing OGC Web Feature and Coverage Service performance: Towards efficient delivery of geospatial data. Journal of Spatial Information Science, 7, 123. doi: http://dx.doi.org/10.5311/JOSIS.2013.7.112.

Hirabayashi, Y., Mahendran, R., Koirala, S., Konoshima, L., Yamazaki, D., Watanabe, S., Kim, H., \& Kanae, S. (2013). Global flood risk under climate change. Nature Climate Change 3, 816-821. doi: 10.1038/nclimate1911.

Martinis, S., Kersten, J., \& Twele, A. (2015). A fully automated TerraSAR-X based flood service. ISPRS Journal of Photogrammetry and Remote Sensing, 104, 203-212. doi: 10.1016/j.isprsjprs.2014.07.014.

OCHA. (2016). Somalia: Humanitarian Snapshot (as of 7 June 2016). Retrieved from http:/ / reliefweb.int/sites/reliefweb.int/files/resources/Somalia $\% 20$ Humanitarian $\% 20$ Snapshot $\%$ 20-\%20June\%202016.pdf .

Pekel, J.F., Cottam, A., Gorelick, N., \& Belward, A.S. (2016). High-resolution mapping of global surface water and its long-term changes. Nature. doi:10.1038/nature20584.

Plate, E.J. (2002). Flood risk and flood management. Journal of Hydrology, 267(1-2), 2-11. doi:10.1016/S0022-1694(02)00135-X.

Sudmanns, M., Tiede, D., Augsten, N., Baraldi, A., Belgiu, M., \& Lang, S. (2016). Array-Datenbanken für semantische inhaltsbasierte Suche und Analyse in Satellitenbildarchiven. Dreiländertagung der DGPF, der OVG und der SGPF in Bern, Schweiz - Publikationen der DGPF 25.

Tiede, D., Baraldi, A., Sudmanns, M., Belgiu, M., \& Lang, S. (2016). ImageQuerying - Earth Observation Image Content Extraction \& Querying across Time and Space, Proc. of the 2016 Conference on Big Data from Space (BiDS'16), 192-195. doi:10.2788/854791.

Twele, A., Cao, W., Plank, S., \& Martinis, S. (2016). Sentinel-1 based flood mapping: a fully-automated processing chain. International Journal of Remote Sensing, 37(13), 2990-3004. doi: http://dx.doi.org/10.1080/01431161.2016.1192304.

Ulaby, F.T., Long, D.G., Blackwell, W.J., Elachi, C., Fung, A.K., Ruf, C., Sarabandi, K., Zebker, H.A., \& Van Zyl, J. (2014). Microwave radar and radiometric remote sensing, 4(5). Ann Arbor: University of Michigan Press. 